\title{
Proper lighting condition for photosynthesis, yield and quality of horticultural crops in controlled environments
}

\author{
(C) Sasan Aliniaeifard \\ Photosynthesis and Light Reactions Laboratory, Department of Horticultural Sciences, \\ Aburaihan Campus, University of Tehran, Iran
}

\section{Email: aliniaeifard@ut.ac.ir}

Keywords: Controlled Environments, Greenhouse Crops, Light, Light Intensity, Light Quality, Photoperiod, Photosynthesis

Light as an energy source for photosynthesis determines the growth, morphology, development and yield of crops. Tendency to produce crops in controlled environments that equipped with artificial light has been increased nowadays. Different characteristics of light including intensity, spectrum and photoperiod are the determining factors for the growth, morphology and yield of crops. In a series of researches done in the photosynthesis and light reactions laboratory of University of Tehran on the role of different lighting conditions on diverse greenhouse crops such as: tomato, lettuce, basil, savory, rose, chrysanthemum, petunia, anthurium, gerbera, carnation, marigold and different types of bromeliads with various photosynthetic metabolisms under controlled environments (e.g. greenhouse, growth chambers and vertical farms), it were shown that different properties of light have significant effects on growth, physiology and yield of the mentioned crops. Furthermore, plant's resistance to various abiotic stresses, including high light intensity, gaseous pollutants, salinity and drought was also affected by growth lighting environment. The results showed that the light spectrum in the range of 600 to $700 \mathrm{~nm}$ increases the growth and yield of most of the mentioned crops and on the other hand the growth of plants exposed to the waveband range of 400 to $500 \mathrm{~nm}$ is often restricted. Despite the positive effects of the light spectrum in the range of 600 to $700 \mathrm{~nm}$ on plant growth, due to the negative effects of this spectrum range on photosynthesis and plant morphology (red light syndrome), lighting environment with red region waveband should be combined with the light spectrum range of 400 to $500 \mathrm{~nm}$. Far red light region of the spectrum caused elongation of internodes. Despite of the negative effects of far red light on the growth and quality of crops, it positively affected the response of plants to stresses. Intensity and quality of light play a significant role in the sensitivity of the plant to diverse abiotic stresses (including drought, gaseous pollutants, salinity and cold). Also, it was found that extending the photoperiod or use of supplemental light on greenhouse crops positively influenced production and quality of the harvested product. The quality of the greenhouse crops was also affected by the spectrum and intensity of the lighting environment. For example, low intensity of ultraviolet light in preharvest stage caused positive impact on postharvest quality of lettuce. Furthermore, application of ultraviolet light on savory plants resulted in an increase in the amount of essential oil. In cut carnation flowers, the best postharvest quality was obtained under blue light, while for plants such as roses and anthuriums (during cold storage), the best quality was obtained under red light or a high percentage of the red light spectral range. Red light by generation of small-sized stomata with narrow aperture restricted the water loss of the leaves. There was a positive relationship between biophysical traits of photosynthesis with postharvest water loss of the leaves that can be used as an index for prediction of postharvest longevity of this flower. In conclusion, it is important to manage lighting condition in the greenhouses and other controlled environments to optimize the production and quality of horticultural crops. 